\title{
Adsorption and Depletion of Polyelectrolytes from Charged Surfaces
}

\author{
Adi Shafir,, , David Andelman,, , \\ ${ }^{1}$ School of Physics and Astronomy \\ Raymond and Beverly Sackler Faculty of Exact Sciences \\ Tel Aviv University, Ramat Aviv, Tel Aviv 69978, Israel \\ ${ }^{2}$ Sektion Physik, Ludwig-Maximilians-Universität \\ Theresienstr. 37, 80333 Munich, Germany
}

(Dated: April 15, 2003)

\begin{abstract}
Mean-field theory and scaling arguments are presented to model polyelectrolyte adsorption from semi-dilute solutions onto charged surfaces. Using numerical solutions of the mean-field equations, we show that adsorption exists only for highly charged polyelectrolytes in low salt solutions. Simple scaling laws for the width of the adsorbed layer and the amount of adsorbed polyelectrolyte are obtained. In other situations the polyelectrolyte chains will deplete from the surface. For fixed surface potential conditions, the salt concentration at the adsorption-depletion crossover scales as the product of the charged fraction of the polyelectrolyte $f$ and the surface potential, while for a fixed surface charge density, $\sigma$, it scales as $\sigma^{2 / 3} f^{2 / 3}$, in agreement with single-chain results.

PACS numbers: 82.35.Gh, 82.35.Rs, 61.41.+e
\end{abstract}

\section{INTRODUCTION}

The phenomenon of adsorption of charged polymer chains (polyelectrolytes) to surfaces has generated a great deal of interest due to its numerous industrial applications and relevance to biological systems. The theoretical treatment is not yet well established because of the multitude of length scales involved, arising from different interactions: electrostatic interactions between monomers and counter-ions, excluded volume interactions and entropic considerations. Furthermore, when salt is added to the solution, the interplay between polyelectrolytes (PEs) and salt ions as well as the ion entropy has to be taken into account.

The adsorption of PE chains onto charged surfaces has been addressed theoretically in several models in the past. They include among others: solutions of linearized mean-field equations 1, 2, 3, 4, 5, 6, 7], numerical solutions of full mean-field equations [8, 9, 10], various scaling theories for single-chain adsorption 11, 12], and formulation of a phenomenological criterion describing the adsorption-depletion transition from charged surfaces 13, 14, 15]. Other approaches employed multiStern layer models 16, 17, 18, where a discrete lattice

*Electronic address: shafira@post.tau.ac.il

${ }^{\dagger}$ Electronic address: andelman@post.tau.ac.il

${ }^{\ddagger}$ Electronic address: netz@theorie.physik.uni-muenchen.de is used and each lattice site can be occupied by either a monomer, a solvent or a small ion. The electrostatic potential can then be calculated self-consistently together with the concentrations of the monomers and counterions.

In this article we re-examine the mean-field equations describing the PE adsorption and their numerical solutions, with specific emphasis on the adsorption-depletion transition. The present paper can be regarded as an extension of Ref. [8]. It agrees with the previously obtained low-salt adsorption regime but proposes a different interpretation of the high-salt regime. We find that the high-salt adsorption regime of Ref. [8] is pre-empted by an adsorption-depletion transition, in analogy with single-chain results. The mean-field equations and their numerical solutions are formulated in Sec. [II some simple scaling relationships in Sec. III] and the adsorptiondepletion transition in Sec. IV] A general discussion and comparison with other models are presented in Sec. $\mathbf{D}$

\section{THE MEAN FIELD EQUATIONS AND THEIR NUMERICAL SOLUTION}

Consider an aqueous solution of infinitely long PEs, together with their counterions and an added amount of salt. Throughout this paper we assume that both the salt ions and counterions are monovalent. Let $\phi(\mathbf{r})=\sqrt{c(\mathbf{r})}$ be the square root of $c(\mathbf{r})$, the local monomer concentra- 
tion, $a$ the monomer size and $f$ the charge fraction on each PE chain. Also let $\phi_{\mathrm{b}}=\sqrt{c_{\mathrm{b}}}$ be the square root of the bulk monomer concentration $c_{\mathrm{b}}$, and $\psi(\mathbf{r})$ the electrostatic potential. The mean-field free energy can be obtained either from phenomenological or field theoretical approaches:

$$
\begin{gathered}
F=\int \mathrm{d} \mathbf{r}\left(f_{\mathrm{pol}}+f_{\mathrm{ion}}+f_{\mathrm{el}}\right) \\
f_{\mathrm{pol}}=k_{\mathrm{B}} T\left(\frac{a^{2}}{6}|\nabla \phi|^{2}+\frac{1}{2} v\left(\phi^{4}-\phi_{\mathrm{b}}^{4}\right)-\mu\left(\phi^{2}-\phi_{\mathrm{b}}^{2}\right)\right) \\
f_{\mathrm{ion}}=k_{\mathrm{B}} T \sum_{i=+,-}\left[c^{i} \ln c^{i}-c^{i}-\mu^{i}\left(c^{i}-c_{\mathrm{b}}^{i}\right)\right] \\
f_{\mathrm{el}}=\left(c^{+}-c^{-}+f \phi^{2}\right) e \psi-\frac{\epsilon}{8 \pi}|\nabla \psi|^{2}
\end{gathered}
$$

While the full details can be found in Refs. [8, 9, 10], here we just briefly explain each of the terms. The first term of $f_{\text {pol }}$ accounts for chain elasticity, the second describes the excluded volume interaction between monomers, where $v$ is the second virial coefficient. The third accounts for the coupling with a reservoir with bulk polymer concentration $\phi_{\mathrm{b}}^{2}=c_{\mathrm{b}}$ and chemical potential $\mu$. The $f_{\text {ion }}$ contribution to the free energy takes into account the entropy of small ions and their chemical potential $\mu^{ \pm}$. Lastly, $f_{\mathrm{el}}$ is the electrostatic free energy. Its first term is the interaction energy between the electrostatic potential and the charged objects; namely, the small ions and monomers. The last term is the self-energy of the electric field $-\frac{\varepsilon}{8 \pi} \int \mathrm{d} \mathbf{r}|\nabla \psi|^{2}$.

Minimizing the free energy with respect to $\psi, \phi, c^{+}, c^{-}$, and using the bulk boundary conditions: $\psi(x \rightarrow \infty)=0$, $\phi(x \rightarrow \infty)=\phi_{\mathrm{b}}, c_{\mathrm{b}}^{-}=c_{\mathrm{salt}}+f \phi_{\mathrm{b}}^{2}$ and $c_{\mathrm{b}}^{+}=c_{\mathrm{salt}}$, the profile equations of Ref. [8] are reproduced:

$$
\begin{gathered}
c^{-}=\left(c_{\text {salt }}+f \phi_{\mathrm{b}}^{2}\right) \mathrm{e}^{\beta e \psi} \\
c^{+}=c_{\text {salt }} \mathrm{e}^{-\beta e \psi} \\
\nabla^{2} \psi=\frac{8 \pi e c_{\text {salt }}}{\varepsilon} \sinh \beta e \psi+\frac{4 \pi e}{\varepsilon}\left(\phi_{\mathrm{b}}^{2} f \mathrm{e}^{\beta e \psi}-f \phi^{2}\right) \\
\frac{a^{2}}{6} \nabla^{2} \phi=v\left(\phi^{3}-\phi_{\mathrm{b}}^{2} \phi\right)+\beta f e \psi \phi,
\end{gathered}
$$

where $\beta=1 / k_{\mathrm{B}} T$ is the inverse of the thermal energy $k_{B} T$. Equations (515) and (6) show that the small ions obey Boltzmann statistics, while Eq. (7) is the Poisson equation where the salt ions, counterions and monomers can be regarded as the sources of the electrostatic potential. Equation (8) is the mean-field (Edwards) equation for the polymer order parameter $\phi(\mathbf{r})$, taking into account the excluded volume interaction and external electrostatic potential $\psi(\mathbf{r})$.

The adsorption onto a flat, homogeneous and charged surface placed at $x=0$ depends only on the distance $x$ from the surface. In this case the above equations can be reduced to two coupled ordinary differential equations. Defining dimensionless variables $\eta \equiv \phi / \phi_{\mathrm{b}}$ and $y \equiv \beta e \psi$, Eqs. (77) and (8) then read:

$$
\begin{aligned}
& \frac{\mathrm{d}^{2} y}{\mathrm{~d} x^{2}}=\kappa^{2} \sinh y+k_{m}^{2}\left(\mathrm{e}^{y}-\eta^{2}\right) \\
& \frac{a^{2}}{6} \frac{\mathrm{d}^{2} \eta}{\mathrm{d} x^{2}}=v \phi_{\mathrm{b}}^{2}\left(\eta^{3}-\eta\right)+f y \eta,
\end{aligned}
$$

where $\kappa^{-1}=\left(8 \pi l_{\mathrm{B}} c_{\text {salt }}\right)^{-1 / 2}$ is the Debye-Hückel screening length, determining the exponential decay of the potential due to the added salt. Similarly, $k_{m}^{-1}=$ $\left(4 \pi l_{\mathrm{B}} \phi_{\mathrm{b}}^{2} f\right)^{-1 / 2}$ determines the exponential decay due to the counterions. The Bjerrum length is defined as $l_{\mathrm{B}}=e^{2} / \varepsilon k_{\mathrm{B}} T$. For water with dielectric constant $\varepsilon=80$, at room temperature, $l_{\mathrm{B}}$ is equal to about $7 \AA$. Note that the actual decay of the electrostatic potential is determined by a combination of salt, counterions, and polymer screening effects.

The solution of Eqs. (9) and (10) requires four boundary conditions. Two of them are the boundary values in the bulk, $x \rightarrow \infty: \eta(x \rightarrow \infty)=1$ and $y(x \rightarrow \infty)=0$, while the other two are the boundary conditions on the $x=0$ surface. In this article we use either constant surface charge density (Neumann boundary conditions) or constant surface potential (Dirichlet boundary conditions). For the former, $\mathrm{d} y /\left.\mathrm{d} x\right|_{x=0}=-4 \pi \sigma e / \varepsilon k_{\mathrm{B}} T=$ $-4 \pi(\sigma / e) l_{\mathrm{B}}$, where $\sigma$ is the surface charge density. For the latter, the surface potential is held fixed with a value: $y(0)=y_{s}$. The other boundary condition for the polymer concentration $\phi$ is taken as a non-adsorbing surface. Namely, $\phi(0)=0$. Note that far from the surface, $x \rightarrow \infty$, Eqs. (92) and (10) already satisfy the boundary condition: $y=0$ and $\phi=\phi_{\mathrm{b}}($ or $\eta=1)$.

Equations (9) and (10) are two coupled non-linear differential equations that do not have a known analytical solution. The numerical solutions of these equations for 
low salt conditions were presented in Ref. [8] and are reproduced here on Figs. 1 and 2, using a different numerical scheme. The numerical results have been obtained using the relaxation method [19] based on a linearization procedure done on a discrete one-dimensional grid. Then, the equations are transformed to a set of algebraic equations for each grid point. The sum of the absolute difference between RHS and LHS over all grid points is minimized iteratively until convergence of the numerical procedure is achieved.

In calculating the numerical profiles of Figs. 1 and 2 we assume positively charged polymers and a constant negative surface potential. In Fig. 1a the reduced electrical potential $y=\beta e \psi$ profile is shown as a function of the distance from the $x=0$ surface. Similarly, in Fig. 2a the monomer rescaled concentration profile $c(x) / c_{\mathrm{b}}$, is shown. In both figures a constant surface potential boundary condition is imposed. The different curves correspond to different surface potentials $y_{s}$, monomer charge fractions $f$ and monomer size $a$. From the numerical profiles of the electrostatic potential and monomer concentration it can be clearly seen that there is a distinct peak in both profiles. Although they do not occur exactly at the same distance from the surface, the corresponding peaks in Fig. 1 and 2 vary in a similar fashion with system parameters. The peak in the concentration (Fig. 2) marks a PE accumulation at the surface and is regarded as a signature of adsorption. The peak in the potential (Fig. 1) marks an over-compensation of surface charges. At the peak of $\psi(x)$, the electric field vanishes, $E=-\mathrm{d} \psi / \mathrm{d} x=0$, meaning that the integrated charge density from the surface up to this distance exactly balances the surface charge.

\section{SCALING ESTIMATE OF THE}

\section{ADSORPTION LAYER: COUNTERION ONLY CASE}

So far numerical solutions within mean-field theory, Eqs. (9) and (10), have been described. We proceed by presenting simplified scaling arguments, which are in agreement with the numerical mean-field results. Note that the treatment here does not capture any correlation effect which goes beyond mean-field. The concept of polymer "blobs" can be useful in order to describe PE adsorption, where such polymer blob can be regarded as a macro-ion adsorbing on a charge surface. The blob size is determined by taking into account the polymer connectivity and entropy as well as the interaction with the charged surface. A single layer of adsorbing blobs is assumed instead of the full continuous PE profile as obtained from the mean-field equations. Therefore, the blob size characterizes the adsorption layer thickness.

\section{A. Fixed Surface Charge Density}

The two largest contributions to the PE adsorption free energy are the electrostatic attraction with the surface and the chain entropy loss due to blob formation.

For simplicity, the electrostatic attraction of the monomers with the surface is assumed to be larger than the monomer excluded volume and monomer-monomer electrostatic repulsion. With this assumption the chain has a Gaussian behavior inside each surface blob, $D \sim$ $a g^{1 / 2}$, where $g$ is the number of monomers in a blob of size $D$, as is shown schematically in Fig. 3. The entropy loss of the chain balances the surface-monomer attraction. As a result the blob attraction with the surface is of order $k_{B} T$. It is now easy to get an estimate of the blob size $D$ :

$$
\begin{array}{r}
f g \frac{\tilde{\sigma}}{\varepsilon} e^{2} D \simeq k_{\mathrm{B}} T \\
D \simeq a g^{1 / 2} \simeq\left(\frac{a^{2}}{l_{\mathrm{B} f \tilde{\sigma}}}\right)^{1 / 3} \\
g \simeq\left(l_{\mathrm{B}} f a \tilde{\sigma}\right)^{-2 / 3},
\end{array}
$$

using a rescaled surface density $\tilde{\sigma} \equiv|\sigma / e|$. These results are in agreement with those describing the statistics of single-chain adsorption [6, 11].

The assumption that the electrostatic attraction to the surface is larger than the monomer-monomer electrostatic repulsion and excluded volume can now be checked self-consistently, yielding two conditions: $\tilde{\sigma} \gg f a^{-2}$ and $f \gg v^{3} /\left(a^{10} \tilde{\sigma} l_{B}\right)$.

The average monomer concentration (per unit volume) in the adsorption layer $c_{m}$, is the blob concentration in the adsorption layer $n_{0}$, times the number of monomers per blob $g$, yielding $c_{m}=n_{0} g$. It is now possible to get an estimate of the blob concentration per unit volume in the adsorption layer, $n_{0}$, by assuming that the adsorbed layer neutralizes the surface charges up to a numerical prefactor of order unity. Hence, $n_{0} \simeq \tilde{\sigma} / D f g$. This assumption, which is in agreement with our numerical 
solutions, leads to

$$
\begin{aligned}
& n_{0} \simeq l_{\mathrm{B}} \tilde{\sigma}^{2} \\
& c_{m}=n_{0} g \simeq\left(l_{\mathrm{B}} a^{-2} f^{-2} \tilde{\sigma}^{4}\right)^{1 / 3} \sim \tilde{\sigma}^{4 / 3} f^{-2 / 3} .
\end{aligned}
$$

Equation (14) is just the Graham equation [21] relating the surface charge density with the counterion density at the surface vicinity. The only difference is that the counterions are replaced by the charged polymer blobs. Furthermore, Eq. (15) is in accord with the results of Ref [14.

The total amount of PEs in the adsorption layer is $\Gamma \simeq$ $c_{m} D=\tilde{\sigma} / f$. In other words, the overall polymer charge in the adsorption layer (up to a numerical prefactor) is $f \Gamma \simeq \tilde{\sigma}$. This is just another way to phrase the charge neutralization by the PEs mentioned above.

\section{B. Fixed Surface Potential}

Using the boundary condition of a fixed surface potential $\psi=\psi_{s}$, the scaling laws for $D$ and $g$ can be obtained in a similar fashion as was done in Sec. IIIA Alternatively, one can (in the absence of salt) relate the surface potential to the surface charge density by $\psi_{s} \simeq \sigma D / \varepsilon$.

The adsorption energy of a blob of charge $g f e$ onto a surface held at potential $\psi_{s}$ is just $g f e \psi_{s}$. Requiring that this energy is of order of $k_{B} T$ we obtain in analogy to Eqs. (11)-(13):

$$
\begin{array}{r}
g f e\left|\psi_{s}\right| \simeq k_{\mathrm{B}} T \\
D \simeq a g^{\frac{1}{2}} \simeq \frac{a}{\sqrt{f\left|y_{s}\right|}} \\
g \simeq \frac{1}{f\left|y_{s}\right|}
\end{array}
$$

Together with the neutralization condition $n_{0} \simeq$ $\tilde{\sigma} /(D f g)$ it yields:

$$
\begin{array}{r}
n_{0} \simeq \frac{f\left|y_{s}\right|^{3}}{l_{\mathrm{B}} a^{2}} \\
c_{m}=n_{0} g \simeq \frac{\left|y_{s}\right|^{2}}{l_{\mathrm{B}} a^{2}} .
\end{array}
$$

Note that the above results are in accord with the ones previously derived in Ref. [8].

Just like in Sec. III.A the self-consistent check can be repeated here for the dominance of the surface-monomer interactions, yielding $\left|y_{s}\right| \gg f^{1 / 3} l_{B}^{2 / 3} a^{-2 / 3}$ and $f \gg$ $v^{2} / a^{6}\left|y_{s}\right|$. This condition has been verified, in addition, by examining numerically the mean-field adsorbing profiles.
The overall charge of the polymer in the adsorbed layer is then:

$$
\begin{aligned}
& \Gamma \simeq c_{m} D \simeq\left|y_{s}\right|^{3 / 2} f^{-1 / 2} l_{\mathrm{B}}^{-1} a^{-1} \sim\left|y_{s}\right|^{3 / 2} f^{-1 / 2} \\
& f \Gamma \simeq\left|y_{s}\right|^{3 / 2} f^{1 / 2} l_{\mathrm{B}}^{-1} a^{-1} \simeq \frac{\left|y_{s}\right|}{l_{\mathrm{B}} D} \simeq\left|\frac{d \psi}{d x}\right|_{x=0} \simeq \tilde{\sigma}
\end{aligned}
$$

which again verifies that the adsorbed amount scales like the surface charge.

The numerical results of the mean-field equations for constant surface potential $y_{s}$ condition and in the low salt regime $\left(c_{\text {salt }}=0.1 \mathrm{mM}\right)$ are consistent with this scaling picture, as can be seen in Figs. 1 and 2. In Fig. 1b the rescaled potential $y /\left|y_{s}\right|$ is plotted in terms of a rescaled distance: $x / D$, with $D$ taken from Eq. (17). In Fig. $2 \mathrm{~b}$ the concentration profile is rescaled by $c_{m}$, Eq. (20), and plotted in terms of the same rescaled distance $x / D$. The figures show clearly data collapse of the two profiles, indicating that the characteristic adsorption length $D$ is indeed given by the scaling predictions. Note that the agreement with the scaling argument occurs as long as the system stays in the low salt limit. The other limit of high salt is discussed next.

\section{THE ADSORPTION - DEPLETION} TRANSITION IN PRESENCE OF ADDED SALT

The same numerical procedure outlined in Sec. II, is used to find when the chains stop adsorbing and instead will deplete from the surface. This is not a sharp transition but rather a crossover which is seen by calculating numerically the PE surface excess, as depicted in Fig. 4. The profiles were obtained by solving numerically the differential equations for several values of $f$ near the adsorption-depletion transition using a fixed surface potential boundary condition. For salt concentration of about $c_{\text {salt }}^{*} \simeq 0.16\left|y_{s}\right| f /\left(l_{\mathrm{B}} a^{2}\right)$ (solid line in Fig. 4), the figure show the disappearance of the concentration peak.

Namely, a depletion-adsorption crossover.

The dependence of $\Gamma=\int_{0}^{\infty} \mathrm{d} x\left(\phi^{2}-\phi_{b}^{2}\right)$ on $c_{\text {salt }}$ and $f$ for constant surface potential is presented in Fig. 5 . The place where $\Gamma=0$ indicates an adsorption-depletion transition, separating positive $\Gamma$ in the adsorption regime from negative ones in the depletion regime. In Fig. $5 \mathrm{a}$ the dependence of $\Gamma$ on $f$ is shown for several salt concentrations ranging from low- to high-salt conditions. For low enough $f, \Gamma<0$ indicates depletion. As $f$ increases, a crossover to the adsorption region, $\Gamma>0$, is seen. In the 
adsorption region, a peak in $\Gamma(f)$ signals the maximum adsorption amount at constant $c_{\text {salt }}$. As $f$ increases further, beyond the peak, $\Gamma$ decreases as $1 / \sqrt{f}$. Looking at the variation of $\Gamma$ with salt, as $c_{\text {salt }}$ increases, the peak in $\Gamma(f)$ decreases and shifts to higher values of $f$. For very large amount of salt, e.g., $c_{\text {salt }}=0.5 \mathrm{M}$, the peak occurs in the limit $f \rightarrow 1$. In Fig. 5 b, we plot $\Gamma\left(c_{\text {salt }}\right)$ for several $f$ values. The adsorption regime crosses over to depletion quite sharply as $c_{\text {salt }}$ increases, signaling the adsorption-depletion transition. The salt concentration at the transition, $c_{\text {salt }}^{*}$, increases with the charge fraction $f$. The dependence of $\Gamma$ on $c_{\text {salt }}$ and $f$ for constant surface charge density is plotted in Fig. 6 . Both salt and $f$ dependences show a similar behaviour to those shown in Fig. 5 for constant surface potential.

The numerical phase diagrams supporting the adsorption-depletion transition are presented in Fig. 7 for constant surface charge conditions. The phase diagrams were obtained by solving numerically the meanfield equations. We scanned the $\left(f, c_{\text {salt }}\right)$ parameter plane for 50 values of $f$ between $0.01<f<1$ (Fig. 7a) and the $\left(\tilde{\sigma}, c_{\text {salt }}\right)$ plane for 50 values of $\tilde{\sigma}=|\sigma / e|$ between $10^{-5}\left[\AA^{-2}\right]<\tilde{\sigma}<10^{-4}\left[\AA^{-2}\right]$ (Fig. 7b). From the loglog plots it can be seen that the adsorption-depletion transition is described extremely well by a line of slope $2 / 3$ in both Fig. $7 \mathrm{a}$ and $7 \mathrm{~b}$. Namely, at the transition $c_{\text {salt }}^{*} \sim f^{2 / 3}$ for fixed $\tilde{\sigma}$ and $c_{\text {salt }}^{*} \sim \tilde{\sigma}^{2 / 3}$ for fixed $f$.

To complete the picture, the adsorption-depletion transition is also presented in Fig. 8 for constant surface potential. The phase diagrams are obtained by solving numerically the differential equations. We scanned the $\left(f, c_{\text {salt }}\right)$ parameter plane for 50 values of $f$ between $0.01<f<1$ (Fig. 8a) and the $\left(\left|y_{s}\right|, c_{\text {salt }}\right)$ plane for 50 values of $\left|y_{s}\right|$ between $0.1<\left|y_{s}\right|<1.0$ (Fig. 8b). From the figure it is apparent that the adsorption-depletion transition line fits quite well a line of slope 1.0 in both Fig. $8 \mathrm{a}$ and $8 \mathrm{~b}$ plotted on a $\log$ - $\log$ scale. Namely, $c_{\text {salt }}^{*} \sim f$ for fixed $y_{\mathrm{s}}$, and $c_{\text {salt }}^{*} \sim y_{\mathrm{s}}$ for fixed $f$.

These scaling forms of $c_{\text {salt }}^{*}$ at the transition can be explained using the simplified scaling arguments introduced in Sec. III.

\section{A. Scaling for Fixed Surface Charge}

If the blobs are taken as charged spheres, the mere existence of an adsorption process requires that the attraction of the monomers to the surface persists for all charges up to distances $D$ from the charged surface. For high ionic strength solutions, the electrostatic potential at distance $x$ for a charged surface can be approximated by the linearized Debye-Hückel potential:

$$
y(x)=4 \pi \tilde{\sigma} l_{\mathrm{B}} \kappa^{-1} \mathrm{e}^{-\kappa x} .
$$

This is valid as long as the potential is low enough, $y \leq$ 1. The adsorption picture requires that the exponential decay of the potential will not vary substantially inside a region of size $D$ comparable to the size of surface blobs, $y(D) \simeq y_{s}$. Then, the exponential decay in Eq. (23) yields

$$
\kappa D<1 .
$$

Namely, the Debye-Hückel screening length is smaller than the adsorption layer thickness, $D$. Using Eq. (12) this yields:

$$
c_{\text {salt }}<\tilde{\sigma}^{2 / 3} f^{2 / 3} l_{\mathrm{B}}^{-1 / 3} a^{-4 / 3}
$$

The crossover between adsorption and depletion will occur when $c_{\text {salt }}^{*} \simeq\left(\tilde{\sigma}^{2} f^{2} l_{\mathrm{B}}^{-1} a^{-4}\right)^{1 / 3}$, in accord with Refs. [6, 7, 12], and with the numerical results discussed above and presented in Fig. 7.

\section{B. Scaling for Fixed Surface Potential}

For the boundary condition, $\psi=\psi_{s}$, the potential decay from the surface can be approximated to be:

$$
y(x)=y_{s} \mathrm{e}^{-\kappa x}
$$

and the same consideration as in Eq. (24) and (25) gives:

$$
c_{\text {salt }}<\frac{\left|y_{s}\right| f}{l_{\mathrm{B}} a^{2}} .
$$

Namely, we expect an adsorption-depletion transition to occur for $c_{\text {salt }}^{*} \simeq\left|y_{s}\right| f /\left(l_{\mathrm{B}} a^{2}\right)$, in the case of a fixed surface potential. This supports the numerical results as presented in Fig. 8.

\section{DISCUSSION}

We have presented numerical calculations of the meanfield equations describing the adsorption of PE chains onto charged surfaces, including multi-chain interactions. The main finding is the existence of an adsorptiondepletion transition in presence of added salt or weakly 
charged chains. The numerical results are discussed in trie. terms of simple scaling arguments describing the adsorption of PEs. The salt concentration at the adsorptiondepletion transition scales like $c_{\text {salt }}^{*} \sim f\left|y_{s}\right|$ for fixed surface potential and $c_{\text {salt }}^{*} \sim(f \tilde{\sigma})^{2 / 3}$ for fixed surface charge density. Within the scaling picture, the condition for depletion is the same as for a single chain, in agreement with our mean-field solutions.

We briefly summarize the main approximations of our mean-field and scaling results. A non-adsorbing surface is used as the polymer boundary condition. However, if the surface has a strong non-electrostatic affinity for the $\mathrm{PE}$ chains, the electrostatic contribution does not have to be the dominant one. The method also assumes Gaussian blobs within mean-field theory. In a more refined theory, excluded volume interactions as well as lateral correlation in the blob-blob interactions will alter the adsorption behavior. When the surface charge (or potential) is high enough, the blob size $D$ can become comparable with the monomer size $a$, and the PE chains will lay flat on the surface. Further investigations might be necessary to address in more detail the above points. It will also be interesting to extend our results to geometries other than the planar charged surface.

Several authors have addressed the problem of adsorption onto surfaces either of a single chain 11] or multiple chains 12] using similar arguments of blobs. In another approach, a Flory-like free energy [8] was introduced using the assumption of a single characteristic length scale. The latter gave adsorption-layer scaling laws as in Eqs. (17) and (20), but did not find the depletion criterion. Instead, an adsorption length scale and a characteristic concentration were predicted for the highsalt regime. We show here, using both numerical calculations and scaling arguments, that the high-salt regime does not exist because it is preempted by a $\mathrm{PE}$ depletion.

Acknowledgments. It is a pleasure to thank G. Ariel, I. Borukhov, Y. Burak, E. Katzav and H. Orland for useful discussions and comments. DA acknowledges support from the Israel Science Foundation under grant No. 210/02 and the Alexander von Humboldt Foundation. RRN acknowledges support from the Deutsche Forschungsgemeinschaft (DFG, GermanFrench Network) and the Fonds der Chemischen Indus- 
[1] R. Varoqui, J. Phys. II (France) 3, 1097 (1993).

[2] R. Varoqui, A. Johner, A. Elaissari, J. Chem. Phys. 94, 6873 (1991).

[3] X. Chatellier and J.F. Joanny, J. Phys. II (France) 6, 1669 (1996).

[4] J.F. Joanny, Eur. Phys. J. B. 9, 117 (2000).

[5] M. Manghi and M. Aubouy, cond-mat/0202045 preprint.

[6] F.W. Wiegel, J. Phys A: Math. Gen. 10, 299 (1977).

[7] M. Muthukumar, J. Chem. Phys. 86, 7230 (1987).

[8] I. Borukhov, D. Andelman, H. Orland, Macromolecules 31, 1665 (1998); Europhys. Lett. 32, 499 (1995).

[9] I. Borukhov, D. Andelman, H. Orland, Eur. Phys. J. B 5, 869 (1998).

[10] I. Borukhov, D. Andelman, H. Orland, J. Phys. Chem. B 103, 5042 (1999).

[11] O.V. Borisov, E.B. Zhulina, T.M. Birshtein, J. Phys. II (France) 4, 913 (1994).

[12] A.V. Dobrynin, A. Deshkovski, M. Rubinstein, Macromolecules 34, 3421 (2001).

[13] R.R. Netz and J.F. Joanny, Macromolecules 32, 9013 (1999).

[14] R.R. Netz and D. Andelman, Neutral and Charged Polymers at Interfaces, to be published, Phys. Rep., 2003.

[15] R.R. Netz and D. Andelman, in: Encyclopedia of Electrochemistry, Eds. M. Urbakh and E. Giladi, Vol. I, (WileyVCH, Weinheim, 2002).

[16] H.A. van der Schee and J. Lyklema, J. Phys. Chem. 88, 6661 (1984).

[17] H.G.M. van de Steeg, M.A. Cohen Stuart, A. de Keizer, B.H. Bijsterbosch, Langmuir 8, 2538 (1992).

[18] M.R. Böhmer, O.A. Evers, J.M.H.M. Scheutjens, Macromolecules 23, 2288 (1990).

[19] W.H. Press, B.P. Flannery, S.A. Teukolsky, W.T. Vetterling, Numerical Recipes in C: The Art of Scientific Computing, (Cambridge University, Cambridge 1992), Chap. 17, p. 762.

[20] D. Andelman, in "Handbook of Biological Physics: Structure and Dynamics of Membranes", Vol. 1B, edited by R.
Lipowsky and E. Sackmann, (Elsevier Science B.V., Amsterdam, 1995), Chap. 12, p. 603.

[21] J.N. Israelachvili, Intermolecular and Surface Forces, (Academic Press, London, 1992). 


\section{Figure Captions}

Fig. 1 (a) Numerical profiles of the rescaled electrostatic potential $y=\beta e \psi$ as function of the distance from the surface $x$ using Eqs. (9) and (10), and constant surface potential. The solid line is for $a=5 \AA$, $f=1, y_{s}=-1.0$, the dotted line for $a=5 \AA, f=$ $1, y_{s}=-0.5$, the dashed line is for $a=10 \AA, f=$ $1, y_{s}=-0.5$, and the dash-dot line for $a=5 \AA, f=$ $0.1, y_{s}=-0.5$. All profiles have $c_{\text {salt }}=0.1 \mathrm{mM}$, $\phi_{\mathrm{b}}^{2}=10^{-6} \AA^{-3}, v=50 \AA^{3}, \varepsilon=80, T=300 \mathrm{~K}$. The profiles reproduce those of Ref. [8] using a different numerical scheme. (b) Same profiles as in part (a) but in rescaled variables: $x / D$ and $y /\left|y_{s}\right|$.

Fig. 2 (a) The concentration profile $c(x) / c_{b}=\phi^{2}(x) / \phi_{\mathrm{b}}^{2}$ for the numerical calculations specified in Fig. 1. The profiles reproduce those of Ref. [8]. (b) Same as in part (a) but in rescaled variables: $x / D$ and $c(x) / c_{m}$.

Fig. 3 A schematic drawing of polyelectrolyte adsorption onto flat surfaces and formation of Gaussian surface blobs each of size $D$ and having $g$ monomers. The monomer size is $a$.

Fig. 4 Numerical polyelectrolyte concentration profiles exhibiting the transition from adsorption to depletion. The dashed line corresponds to $f=0.12$, the dot-dash line to $f=0.1$, the solid line to $f=0.09$, and the dotted line to $f=0.08$. All profiles have $\left|y_{s}\right|=0.5, \phi_{b}^{2}=10^{-6} \AA^{-3}, v=50 \AA^{3}, a=5 \AA$, $c_{\text {salt }}=70 \mathrm{mM}$. The adsorption-depletion transition is found to occur for $f=0.09$, corresponding to $c_{\text {salt }}^{*} \simeq 0.16\left|y_{s}\right| f / l_{B} a^{2}$.

Fig. 5 (a) Surface excess of PE adsorption, $\Gamma$, as function of the chain charged fraction $f$, for several salt concentrations: $1.0 \mathrm{mM}$ (solid line), $10 \mathrm{mM}$ (dashed line), $0.1 \mathrm{M}$ (dash-dot line), $0.5 \mathrm{M}$ (dots), and for constant surface potential. As the salt concentration increases, the peak in $\Gamma$ shifts to higher $f$ values and disappears for $c_{\text {salt }}=0.5 \mathrm{M}$. The depletionadsorption transition occurs for $\Gamma=0$. (b) Surface excess as function of salt concentration, $c_{\text {salt }}$, for several $f$ values: $\mathrm{f}=0.03$ (dots), 0.1 (dashes), 0.3 (dot-dash), 1.0 (solid line). $\Gamma$ is almost independent of $c_{\text {salt }}$ for low salt concentrations in the adsorption region. It is then followed by a steep descent into a depletion region at a threshold value.
Other parameters used are: $y_{s}=-1.0, v=50 \AA^{3}$, $\phi_{b}^{2}=10^{-6} \AA^{-3}, a=5 \AA, T=300 \mathrm{~K}$ and $\varepsilon=80$.

Fig. 6 (a) Surface excess of PE adsorption, $\Gamma$, as function of the chain charged fraction $f$, for several salt concentrations: $4.0 \mathrm{mM}$ (solid line), $8.0 \mathrm{mM}$ (dashed line), $21 \mathrm{mM}$ (dash-dot line), $63 \mathrm{mM}$ (dots), and for constant surface charge density. As the salt concentration increases, the peak in $\Gamma$ shifts to higher $f$ values and disappears for $c_{\text {salt }}=63 \mathrm{mM}$. The depletion-adsorption transition occurs for $\Gamma=0$. (b) Surface excess as function of salt concentration, $c_{\text {salt }}$, for several $f$ values: $\mathrm{f}=0.1$ (dots), 0.2 (dashes), 0.45 (dot-dash), 1.0 (solid line). $\Gamma$ is almost independent of $c_{\text {salt }}$ for low salt concentrations in the adsorption region. It is then followed by a steep descent into a depletion region at a threshold value. Other parameters used are: $\sigma / e=-10^{-4} \AA^{-2}, v=50 \AA^{3}, \phi_{b}^{2}=10^{-6} \AA^{-3}$, $a=5 \AA, T=300 \mathrm{~K}$ and $\varepsilon=80$.

Fig. 7 Numerically calculated adsorption-depletion crossover diagram for constant surface charge condition. In (a) the $\left(f, c_{\text {salt }}\right)$ parameter plane on a log-log scale while $\tilde{\sigma}=|\sigma / e|$ is held constant at $\tilde{\sigma}=10^{-3} \AA^{-2}$. The full squares represent the lowest salt concentration for which depletion is detected. The least-mean-square fit to the data points gives a straight line with slope of $0.69 \pm 0.02$. The figure shows that the numerical results agree with a $2 / 3$ power law as predicted in Sec. IV.A, $c_{\text {salt }}^{*} \sim f^{2 / 3}$. In (b) the crossover diagram in calculated numerically in the $\left(|\sigma / e|, c_{\text {salt }}\right)$ parameter plane on a log-log scale, while $f$ is fixed to be $f=0.1$ The least-mean-square line has a slope of $0.71 \pm 0.02$, showing that the numerical results agree with a $2 / 3$ power law as predicted in Sec. IV.A, $c_{\text {salt }}^{*} \sim \sigma^{2 / 3}$.

Fig. 8 Numerically calculated crossover diagram on a loglog scale for constant surface potential conditions. Notations and symbols are the same as in Fig. 6 . In (a) the $\left(f, c_{\text {salt }}\right)$ parameter plane is presented for constant $y_{\mathrm{s}}=-1.0$. The least-mean-square fit has a slope of $1.00 \pm 0.02$, in excellent agreement with the scaling arguments, $c_{\text {salt }}^{*} \sim f$. In (b) the $\left(\left|y_{s}\right|, c_{\text {salt }}\right)$ parameter plane is presented, for constant $f=0.1$. The least-mean-square fit has a slope of $1.04 \pm 0.02$, in agreement with scaling arguments, $c_{\text {salt }}^{*} \sim\left|y_{s}\right|$. 

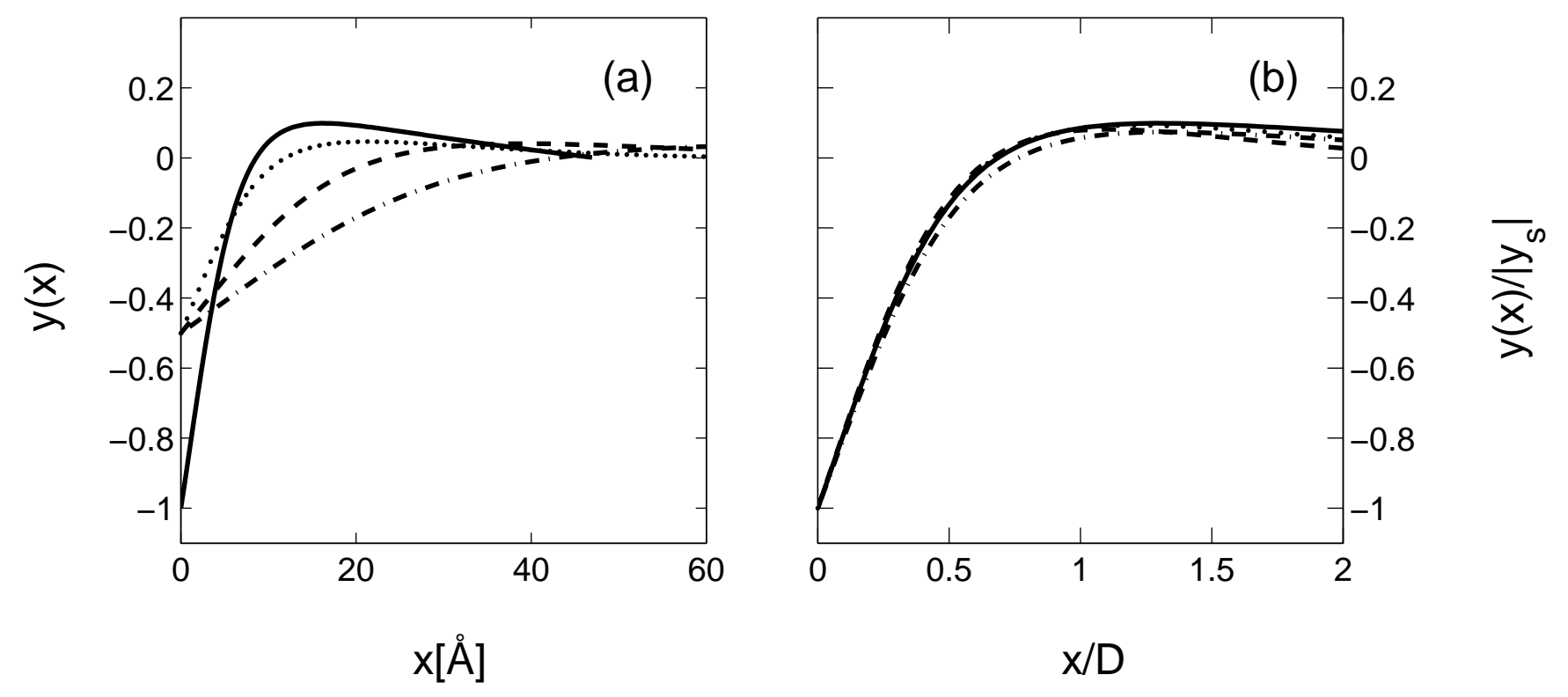

Fig. 1, Shafir et al
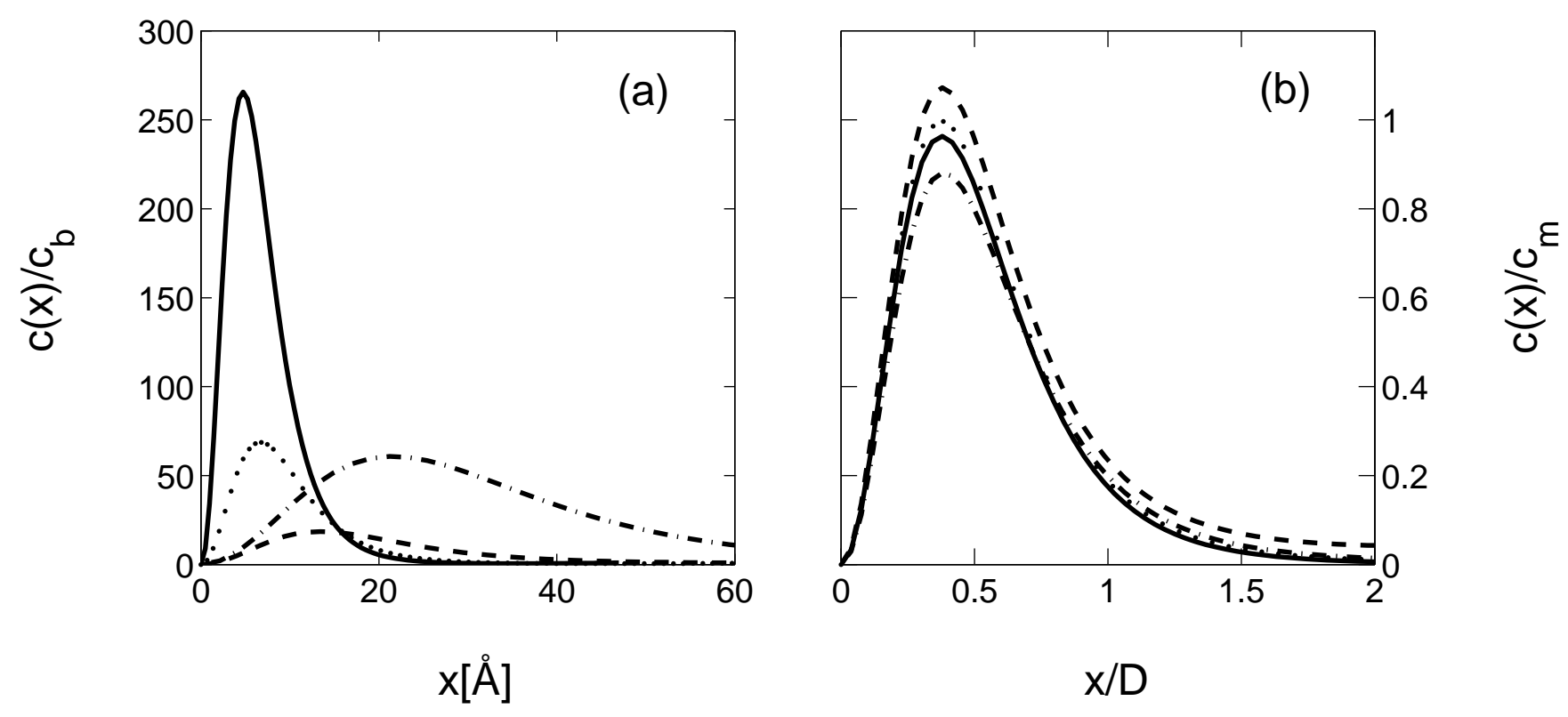

Fig. 2, Shafir et al 


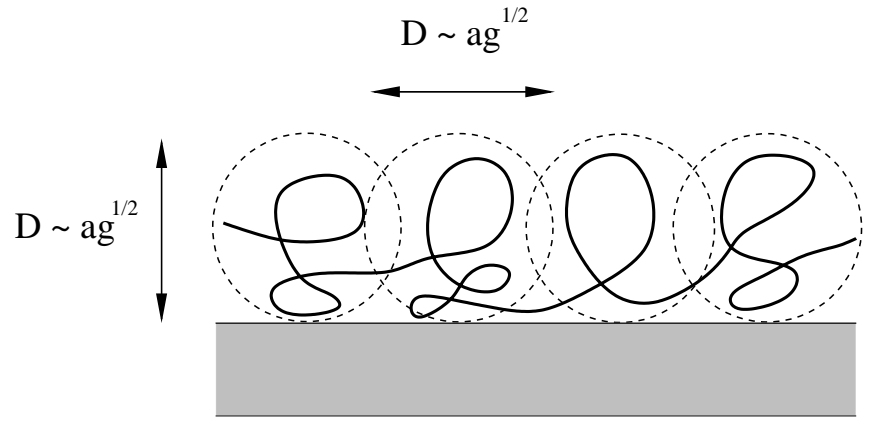

Fig. 3, Shafir et al

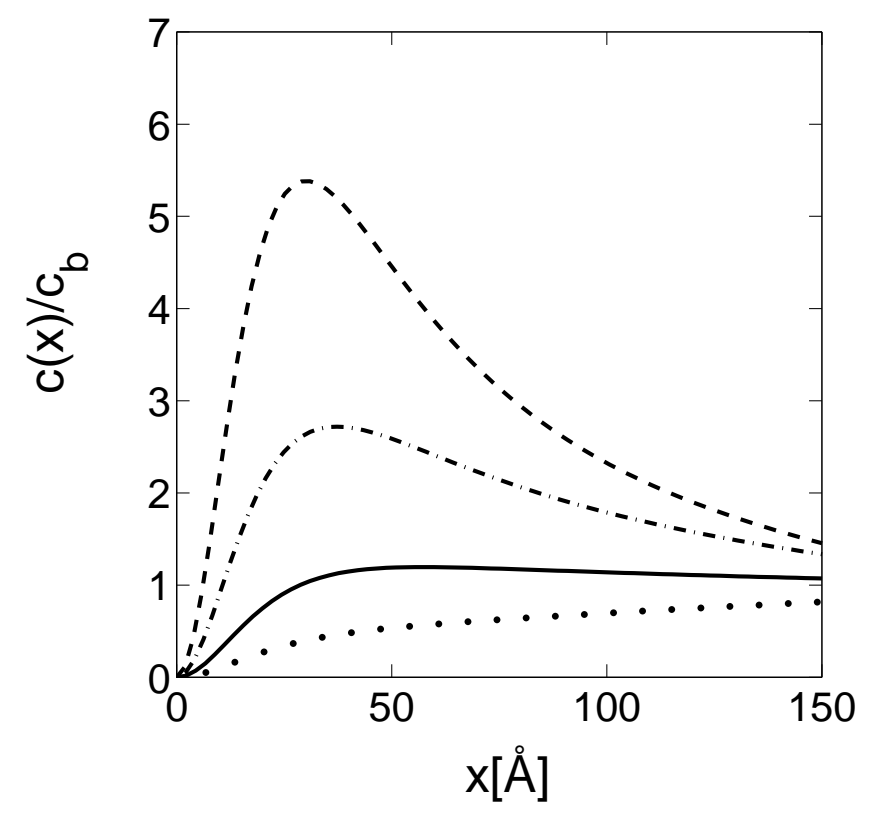

Fig. 4, Shafir et al 

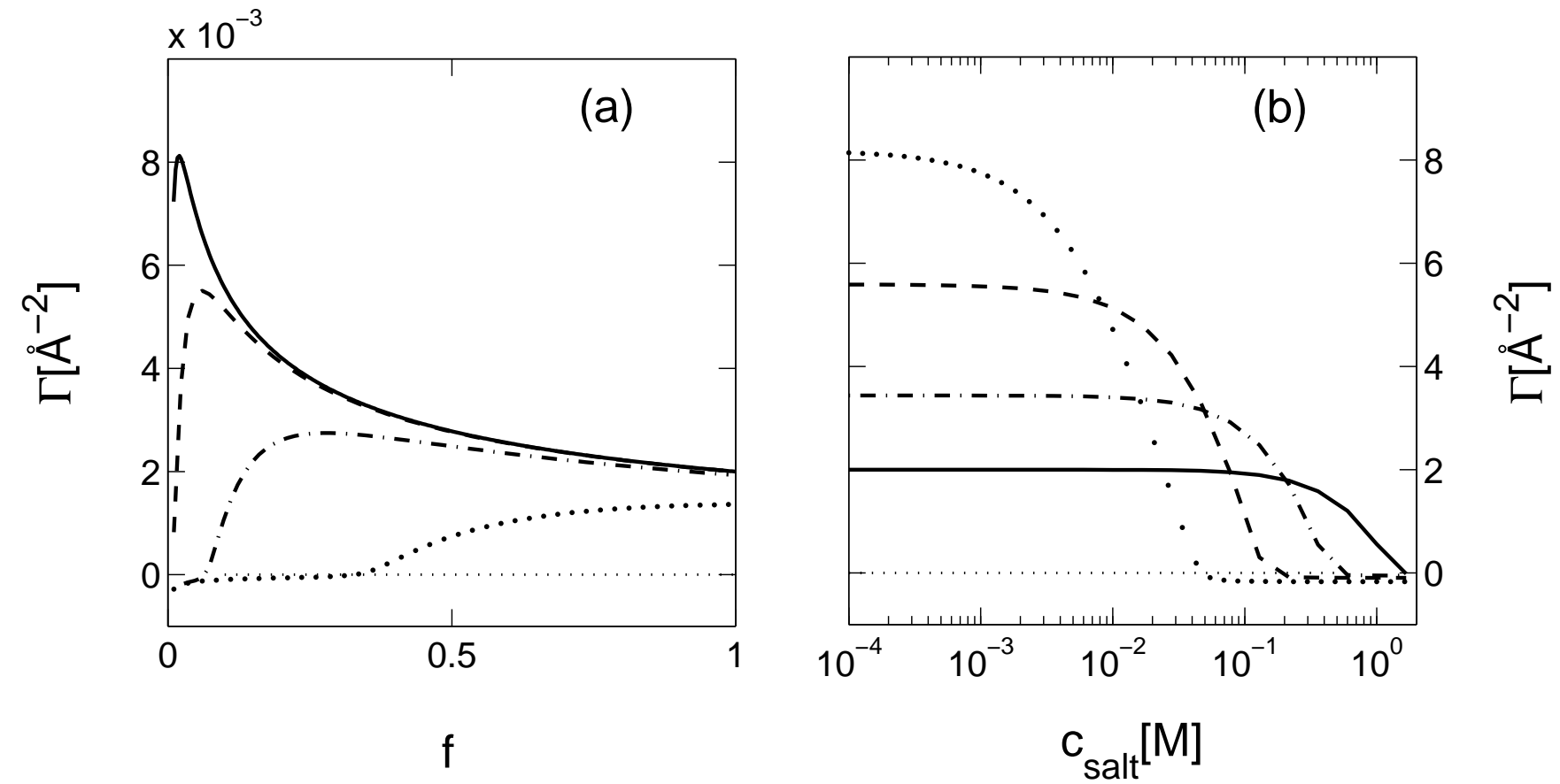

Fig. 5, Shafir et al
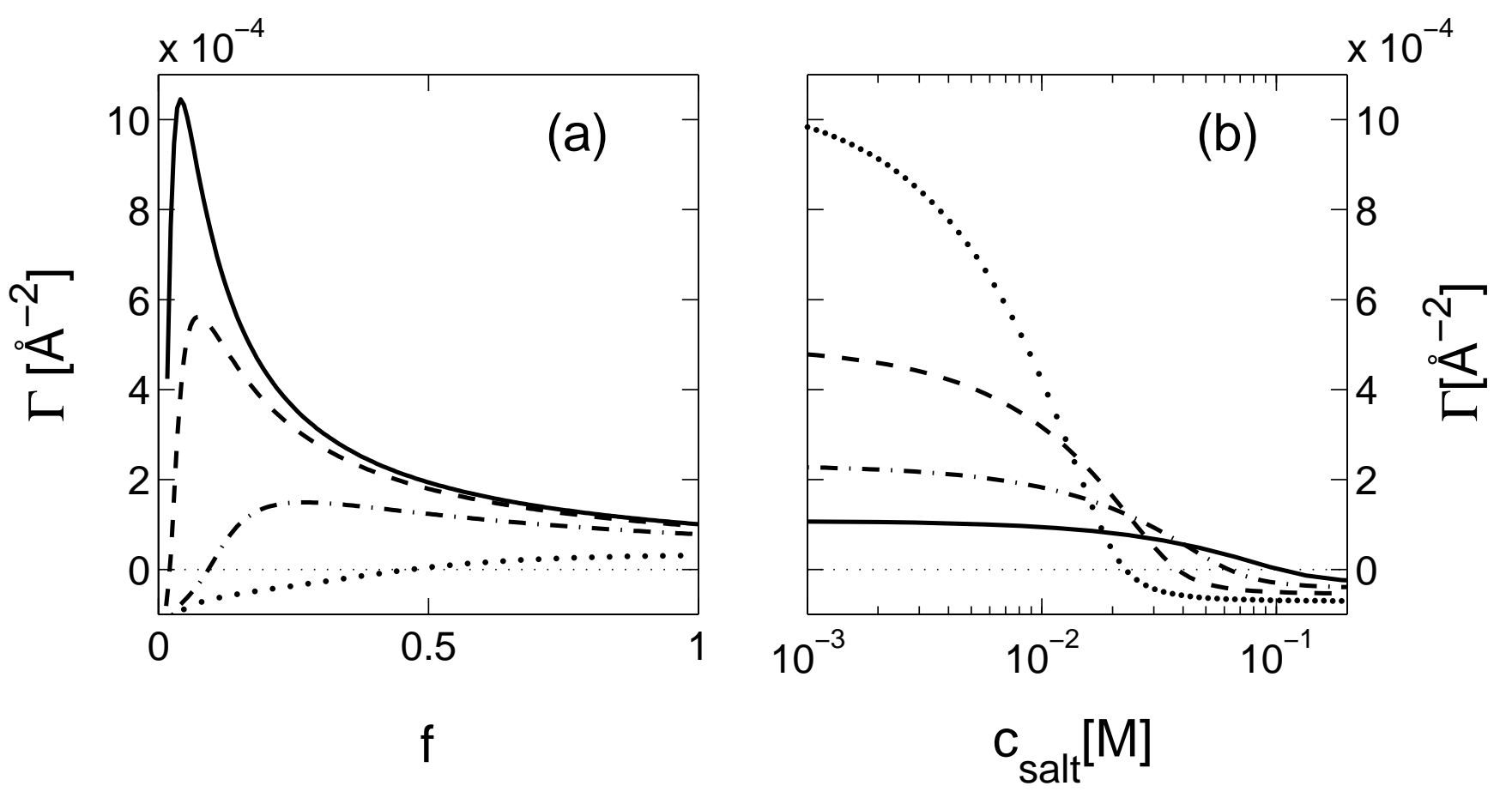

Fig. 6, Shafir et al 

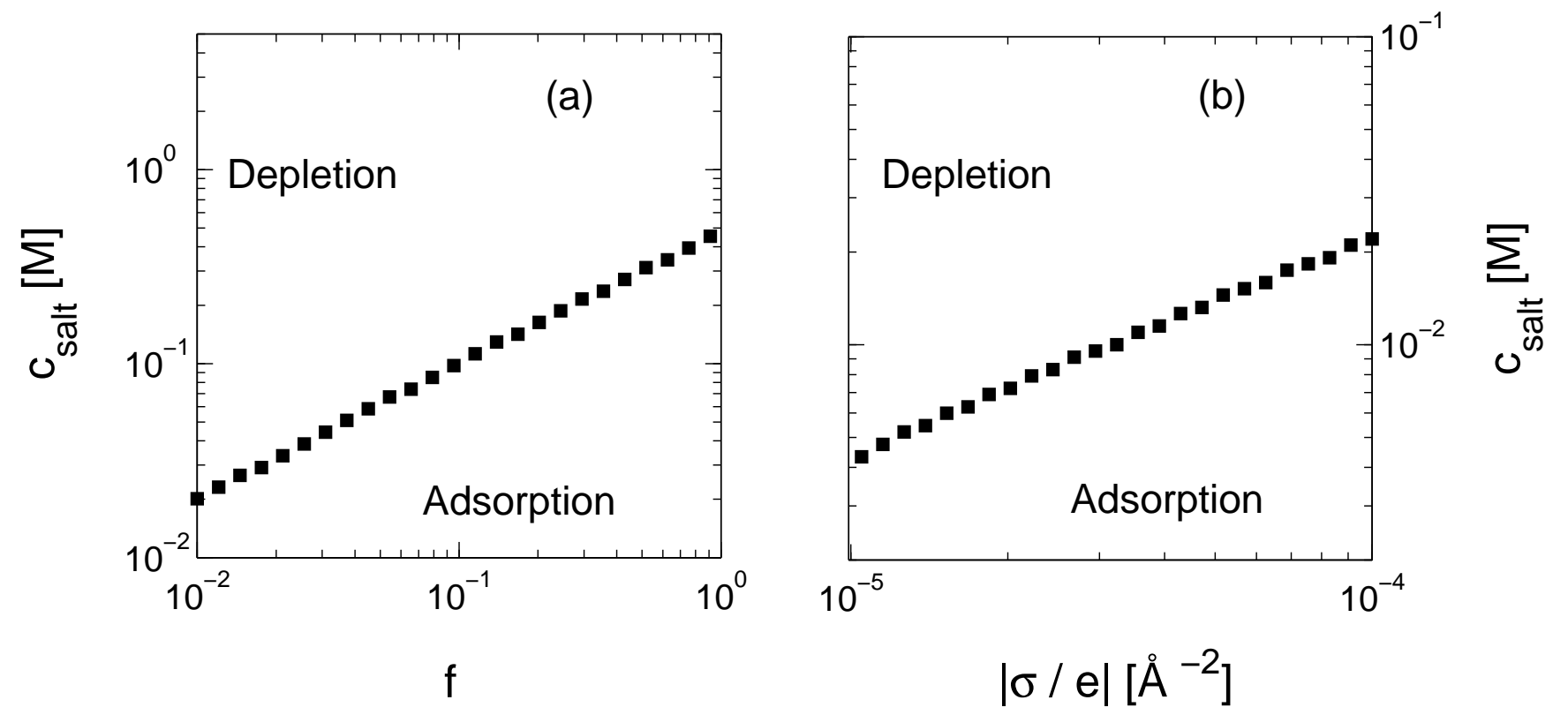

Fig. 7, Shafir et al
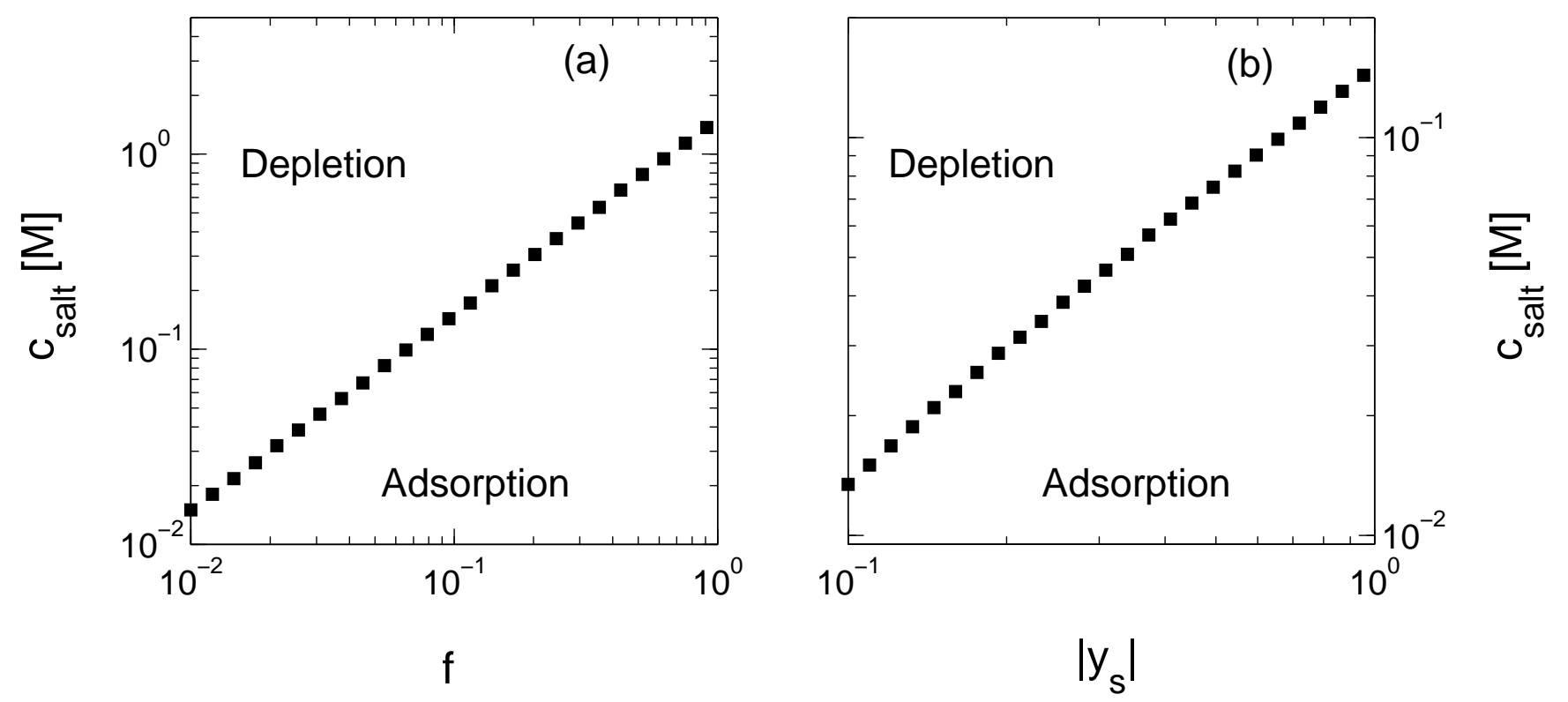

Fig. 8, Shafir et al 